\title{
Beaming of light and enhanced transmission via surface modes of photonic crystals
}

\author{
Irfan Bulu, Humeyra Caglayan, and Ekmel Ozbay \\ Nanotechnology Research Center and Department of Physics, Bilkent University, Ankara 06800, Turkey
}

Received June 6, 2005; revised manuscript received July 21, 2005; accepted July 21, 2005

We report beaming and enhanced transmission of electromagnetic waves by use of surface corrugated photonic crystals. The modes of a finite-size photonic crystal composed of dielectric rods in free space have been analyzed by the plane-wave expansion method. We show the existence of surface propagating modes when the surface of the finite-size photonic crystal is corrugated. We theoretically and experimentally demonstrate that the transmission through photonic crystal waveguides can be substantially increased by the existence of surface propagating modes at the input surface. In addition, the power emitted from the photonic crystal waveguide is confined to a narrow angular region when an appropriate surface corrugation is added to the output surface of the photonic crystal. (C) 2005 Optical Society of America

OCIS codes: $240.0240,050.0050$.

Photonic crystals (PCs) are artificially designed periodic dielectric materials. ${ }^{1}$ The periodic index modulation leads to strong modification of the dispersion diagram for the electromagnetic (EM) waves. The strong modification of the dispersion diagram has been one of the major reasons behind the extensive research on PCs in the past decade. Many interesting applications based on PCs have been proposed and demonstrated. Waveguides ${ }^{2}$ and highly directional antennas ${ }^{3}$ are among these applications.

A metallic surface and the surface of a corrugated PC have in common that both surfaces can support surface-propagating EM waves. ${ }^{4,5}$ The properties of surface-propagating modes on metallic surfaces have been extensively studied. Recently, extraordinary light transmission through a subwavelength aperture on a metallic surface surrounded by concentric grooves or a grating was demonstrated., ${ }^{6,7}$ This extraordinary transmission is attributed to the excitation of surface modes on the metallic surface. In addition to the extraordinary transmission, beaming of EM waves via surface modes on the metallic surfaces has been reported. ${ }^{8}$ Moreno et al. ${ }^{9}$ theoretically demonstrated that similar effects such as enhanced transmission through a PC waveguide and beaming of EM waves can be observed by using PCs. Moreover, Kramper et al. ${ }^{10}$ demonstrated a beaming effect in optical regions by using PC structures.

In this paper, we experimentally demonstrate high transmission amplitudes through PC waveguides when a surface corrugation is added to the input surface of the PC. In addition, our far-field radiation pattern measurements show that, when a periodic corrugation is added to the output surface of the $\mathrm{PC}$, the power emitted through the $\mathrm{PC}$ waveguide is confined to a narrow angular region.

Modes of an infinite PC can be calculated by using the plane-wave expansion method. ${ }^{11}$ The same method can be used to identify the modes of a finitesize PC by employing a large enough supercell. We study TM-polarized (electric field parallel to the axis of the rods) EM waves throughout this work. The PC that we used in our study is a two-dimensional square array of circular alumina rods. The radius of the rods is $1.55 \mathrm{~mm}$. The dielectric constant of alumina is 9.61 , and the lattice constant is $11 \mathrm{~mm}$. The supercell has a rectangular geometry, and it consists of 40 layers long along the $y$ axis and 1 unit cell along the $x$ axis; 15 unit cells along the $y$ axis contain alumina rods, and the rest is free space. We calculated the band structure of an infinite PC [Fig. 1(a)] and the finite-size PC [Fig. 1(b)] that we used in our experimental studies. The modes of a finite-size PC (infinitely periodic along one of the axes and finite in the other axis) can be classified into three parts: (1) modes extending both in air and in the PC, (2) modes extending in air but decaying in the PC, (3) modes decaying in air but extending in the PC. ${ }^{4}$ Only the modes that extend both in air and in the PC are observed in the transmission spectrum.

The PC surface supports surface propagating waves when an appropriate corrugation is added. ${ }^{4}$ The corrugation may be achieved by reducing the rod radius at the surface of the $\mathrm{PC}$ or by using rods of different shape. For this study we changed the radius of the rods at the surface of the PC from 1.55 to $0.76 \mathrm{~mm}$. The band structure of the corresponding PC is calculated by the plane-wave expansion method with a large supercell [Fig. 1(c)]. Figure

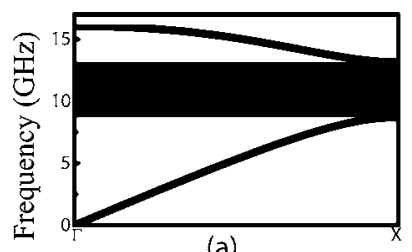

(a)

(c)

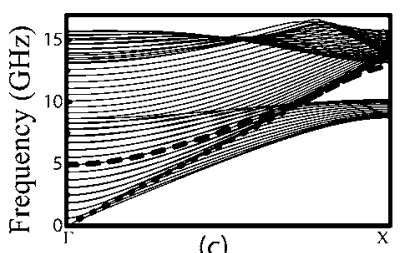

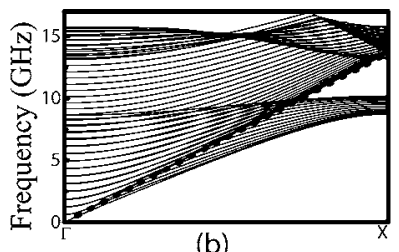

(b)

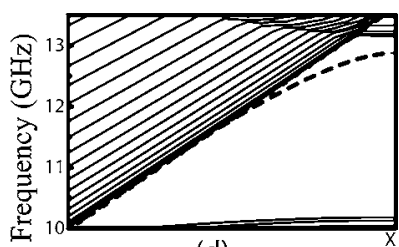

(d)
Fig. 1. (a) TM (electric field parallel to the axis of the rods) band structure of the infinite-size PC. (b) Band structure of the finite-size PC. (c) Band structure of the finite-size PC when the corrugation is added. (d) Enlarged view of (c). 


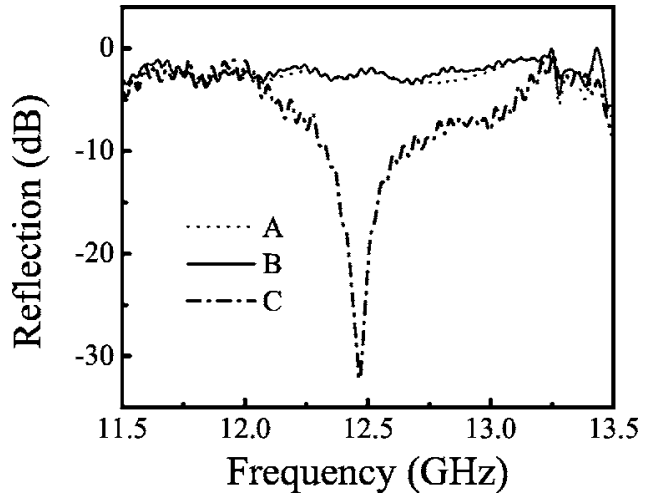

Fig. 2. Measured reflection spectrum from (curve A) the bare PC surface, (B) from the PC surface with an added corrugated layer, (C) from the corrugated PC surface when the gratinglike structure is added.

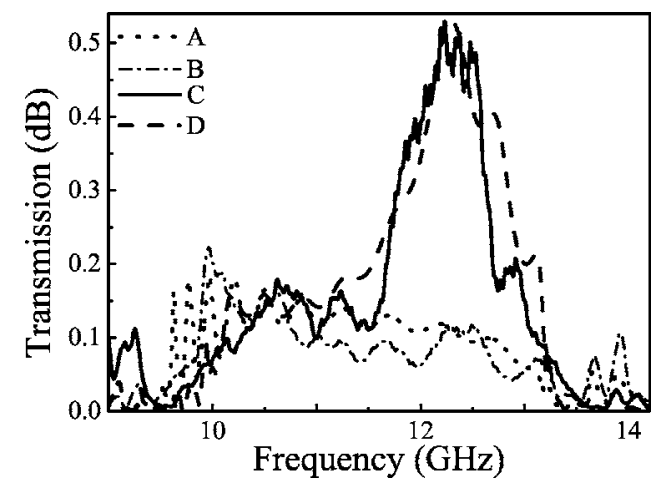

Fig. 3. Curve A, measured and B, calculated transmission spectrum through the PC waveguide; curve $\mathrm{C}$, measured and, D, calculated transmission spectrum through the PC waveguide when the surface corrugation and the gratinglike structure is added in front of the input surface of the $\mathrm{PC}$ waveguide.

1(c) shows that when the corrugation is added a band below the light line appears in the band structure. [This band is shown with a dashed curve in Figs. 1(c) and 1(d).] This band is inside the photonic bandgap and extends from 11.9 to $12.8 \mathrm{GHz}$. The electric field for these modes is evanescent both in air and inside the PC. However, these modes have real wave vectors parallel to the PC surface. As a result, these modes are surface-propagating waves.

Since the surface-propagating modes lie below the light line [Fig. 1(c)], they cannot be excited by incident plane waves. However, these modes can be excited by adding a gratinglike structure to the surface of the corrugated PC, i.e., by adding an extra layer with a suitable lattice constant. The added layer is composed of alumina rods with a radius of $1.55 \mathrm{~mm}$ and a lattice constant of $22 \mathrm{~mm}$. The coupling to the surface modes of the PC can then be observed by measuring the reflection spectrum. We measured the reflection spectrum by using an HP-8510C vector network analyzer and transmitting-receiving horn antennas. The measured reflection spectrum is shown in Fig. 2. Both the uncorrugated and the corrugated PC effectively reflect the incident EM waves. On the other hand, when the gratinglike layer is added to the surface of the corrugated PC, the resulting struc- ture exhibits a dip in the reflection spectrum around $12.4 \mathrm{GHz}$. The magnitude of the reflection coefficient is $-32 \mathrm{~dB}$ at $12.4 \mathrm{GHz}$. The dip in the reflection spectrum clearly shows that because of the gratinglike structure the incident EM waves effectively couple to the surface modes of the corrugated PC around $12.4 \mathrm{GHz}$. If one calculates the coupled surface mode wavelength from the period of the gratinglike layer, $\lambda_{\text {surface }}=$ Period $_{\text {gratinglike layer, this frequency for our }}$ structure turns out to be $13.6 \mathrm{GHz}$. On the other hand, the measured value is around $12.4 \mathrm{GHz}$. The discrepancy may be attributed to the effect of the basis and the unit cell that make the gratinglike layer. Hence, the geometry and the content of the gratinglike layer must be taken into account.

Important components of photonic devices include the waveguides. Previously, several waveguide structures based on PCs have been proposed and demonstrated. ${ }^{2}$ PC-based waveguides are usually fabricated either by removing a complete row from the PC or by creating coupled defects. One major problem with PC-based waveguides is low transmission efficiency.

Recently, enhanced transmission through subwavelength metallic apertures surrounded by concentric grooves or a grating was demonstrated by several researchers. ${ }^{6,7}$ The concentric grooves or the grating is used to excite the surface-propagating modes on the metal. It has been suggested that surface modes play an important role in the enhanced transmission.

We fabricated a PC waveguide by removing one row of rods from a $21 \times 15$ square array of circular alumina rods. The crystal is 15 layers long along the propagation direction. The measured and the calculated spectra transmitted through the PC waveguide are shown in Fig. 3. A waveguide band between 9.7 and $13.1 \mathrm{GHz}$ is observed in the transmission spec-
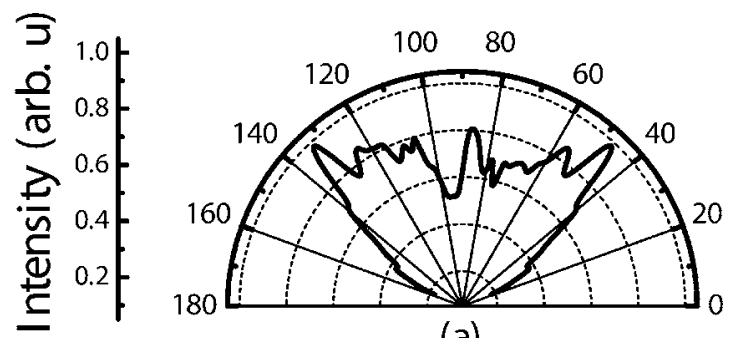

(a)
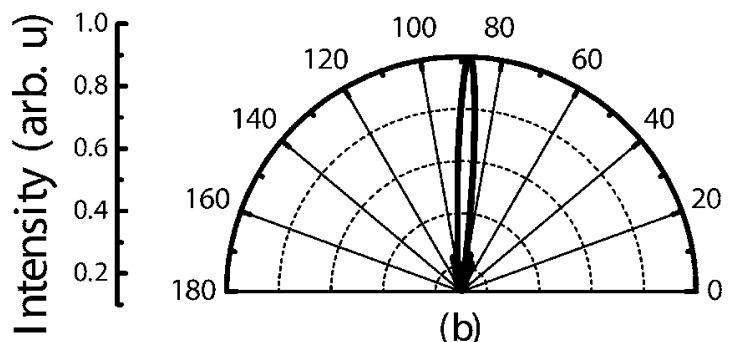

(b)

Fig. 4. (a) Measured far-field radiation pattern of the EM waves emitted from the PC waveguide at $12.45 \mathrm{GHz}$. (b) Measured far-field radiation pattern of EM waves emitted from the PC waveguide with surface corrugation and a gratinglike layer. 


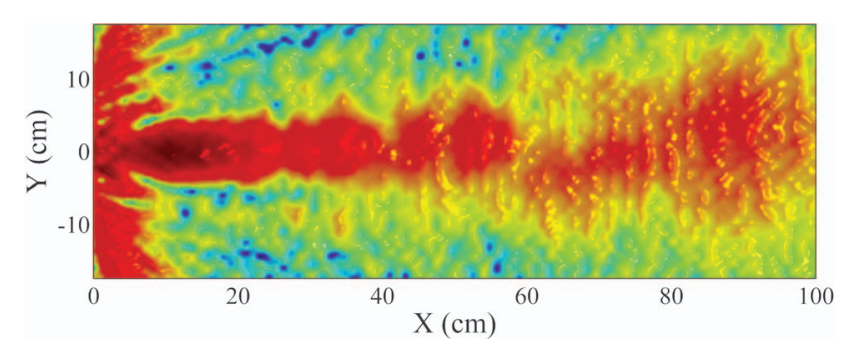

Fig. 5. Measured intensity distribution at the exit side of the PC waveguide when the corrugation and gratinglike layer are added to the exit surface of the PC waveguide. The $Y$ axis is parallel to the PC surface.

trum of the PC waveguide. The overall transmission efficiency is around $10 \%$ within the waveguide band compared with free-space transmission.

We added an extra layer of rods with a radius of $0.76 \mathrm{~mm}$ to the input surface of the PC. As we have shown previously, addition of this extra layer creates surface modes. In order to couple to the surface modes, we added the gratinglike layer in front of the extra layer. Note that the gratinglike layer results in efficient coupling to the surface-propagating modes around $12.45 \mathrm{GHz}$ (Fig. 2). The measured and calculated spectra transmitted through the PC waveguide with the input surface modulation are shown as curves $\mathrm{C}$ and $\mathrm{D}$ in Fig. 3. The transmission efficiency is increased by a factor of 5 around $12.45 \mathrm{GHz}$ when compared with the bare $\mathrm{PC}$ waveguide. These results indicate that efficient coupling to the $\mathrm{PC}$ waveguide modes can be achieved via surface propagating waves.

The EM waves emitted through a subwavelength aperture quickly diffract in all directions. ${ }^{12}$ Recently, it was shown that when a subwavelength aperture on a metal surface is dressed with a grating or concentric grooves, the EM waves emerging from the subwavelength aperture are confined to a narrow spatial region. ${ }^{8}$ This phenomenon is usually called beaming.

The PC waveguide that we used in this study has a width smaller than the operation wavelength. The operation wavelength is around $2.5 \mathrm{~cm}$, whereas the waveguide width is $1.9 \mathrm{~cm}$. Hence the EM waves emitted through the PC waveguide would diffract in all directions from the PC waveguide aperture. The far-field radiation pattern of the waves emitted through the PC waveguide is shown in Fig. 4(a). Figure 4(a) shows that the emitted power spreads into a wide angular region.

The surface modes can be excited by the PC waveguide. The excited surface modes can be coupled to the radiating modes of free space when a gratinglike layer is added in front of the corrugation. The measured far-field radiation patterns of the EM waves emitted from the PC waveguide with surface corrugation and the gratinglike layer are shown in Fig. 4(b). Figure 4(b) shows that with the surface corrugation and the gratinglike layer of the emitted power are confined to a very narrow angular region with a half-power beam width of $10^{\circ}$. In addition, the measured electric field intensity at $12.45 \mathrm{GHz}$ over a $35 \mathrm{~cm} \times 50 \mathrm{~cm}$ area on the exit side of the PC waveguide in the presence of corrugation and the gratinglike layer is shown in Fig. 5. Figure 5 shows that the electric field intensity is confined to a narrow spatial region and propagates without diffracting into a wide angular region.

In conclusion, we have demonstrated beaming and enhanced transmission of EM waves by using surface modes of corrugated PC structures. The measured transmission of a PC waveguide structure without surface corrugation was about $10 \%$. The transmission increased to $55 \%$ when a surface corrugation with a gratinglike layer was added to the input surface of the PC waveguide. In addition, we demonstrated that the electric field emitted through the PC waveguide in the presence of the surface corrugation and the gratinglike layer was confined to a narrow spatial region.

This work was supported by the European Union under the projects EU-DALHM, EU NOEMETAMORPHOSE, EU-NOE-PHOREMOST, and TUBITAK. E. Ozbay acknowledges partial support from the Turkish Academy of Sciences. I. Bulu's e-mail address is irfan@fen.bilkent.edu.tr.

\section{References}

1. E. Yablonovitch, Phys. Rev. Lett. 58, 2059 (1987).

2. S. G. Johnson, P. R. Villeneuve, S. Fan, and J. D. Joannopoulos, Phys. Rev. B 62, 8212 (2000).

3. I. Bulu, H. Caglayan, and E. Ozbay, Appl. Phys. Lett. 83, 3263 (2003).

4. R. D. Meade, K. D. Brommer, A. M. Rappe, and J. D. Joannopoulos, Phys. Rev. B 44, 10961 (1991).

5. D. Beaglehole, Phys. Rev. Lett. 22, 498 (1967).

6. T. Thio, H. J. Lezec, T. W. Ebessen, K. M. Pellerin, G. D. Lewen, A. Nahata, and R. A. Linke, Nanotechnology 13, 429 (2002).

7. H. Caglayan, I. Bulu, and E. Ozbay, Opt. Express 13, 1666 (2005).

8. H. J. Lezec, A. Degiron, E. Devaux, R. A. Linke, L. Martin-Moreno, F. J. Garcia-Vidal, and T. W. Ebbesen, Science 297, 820 (2002).

9. E. Moreno, F. J. Garcia-Vidal, and L. Martin-Moreno, Phys. Rev. B 69, 121402 (2004).

10. P. Kramper, M. Agio, C. M. Soukoulis, A. Birner, F. Muller, R. B. Wehrspohn, U. Gosele, and V. Sandoghdar, Phys. Rev. Lett. 92, 113903 (2004).

11. K. M. Ho, C. T. Chan, and C. M. Soukoulis, Phys. Rev. Lett. 65, 3152 (1990).

12. H. A. Bethe, Phys. Rev. 66, 163 (1944). 